\title{
Silicon Quantum Dots: A General Photocatalyst for Reduction, Decomposition, and Selective Oxidation Reactions \\ (Supporting information)
}

\section{Experiment and characterization details}

The chemicals were purchased from Sigma-Aldrich and Beijing Chemical Reagent (Beijing, China). Each solution of hydrogen peroxide $\left(\mathrm{H}_{2} \mathrm{O}_{2}\right)$ was freshly prepared immediately before use. Other reagents were analytical-grade and used as received. The TEM image and ED pattern of SiQDs were obtained with a FEI/Philips Techal 12 BioTWIN TEM and JEM-2010 transmission electron microscope. The PL study of SiQDs was performed with a RENISHAW UV micro-Raman spectrometer (excitation wavelength being $244 \mathrm{~nm}$ ). Hydroxylation products were analyzed using a gas chromatograph and GC/MS Chemstation system: Hewlett-Packard 6890/5793 GC-MS equipped with a HP 5MS column (30 m long, $0.25 \mathrm{~mm}$ i.d., $0.25 \mu \mathrm{m}$ film thickness). The gas chromatography was performed in a Varian $3400 \mathrm{GC}$ column with a crosslinked 5\% PhMe silicone column $(25 \mathrm{~m} \times 0.20 \mathrm{~mm} \times 0.33 \mu \mathrm{m})$ and a FID detector with the following conditions: Carrier gas $\left(\mathrm{N}_{2}\right)$ at $140 \mathrm{Kp}$; temperature program $60^{\circ} \mathrm{C}, 1 \mathrm{~min}, 15^{\circ} \mathrm{C} / \mathrm{min}, 200^{\circ} \mathrm{C}, 15 \mathrm{~min}$; Split ratio, $10: 1$; Injector, $300^{\circ} \mathrm{C}$; detector, $300^{\circ} \mathrm{C}$. The concentration of formaldehyde and methyl red was monitored by Aglient 8453 UV-Vis Diode Array Spectrophotometer. ${ }^{1} \mathrm{H}-\mathrm{NMR}$ was performed with Varian mercury plus $300\left(\mathrm{CDCl}_{3}\right.$ as solvent).

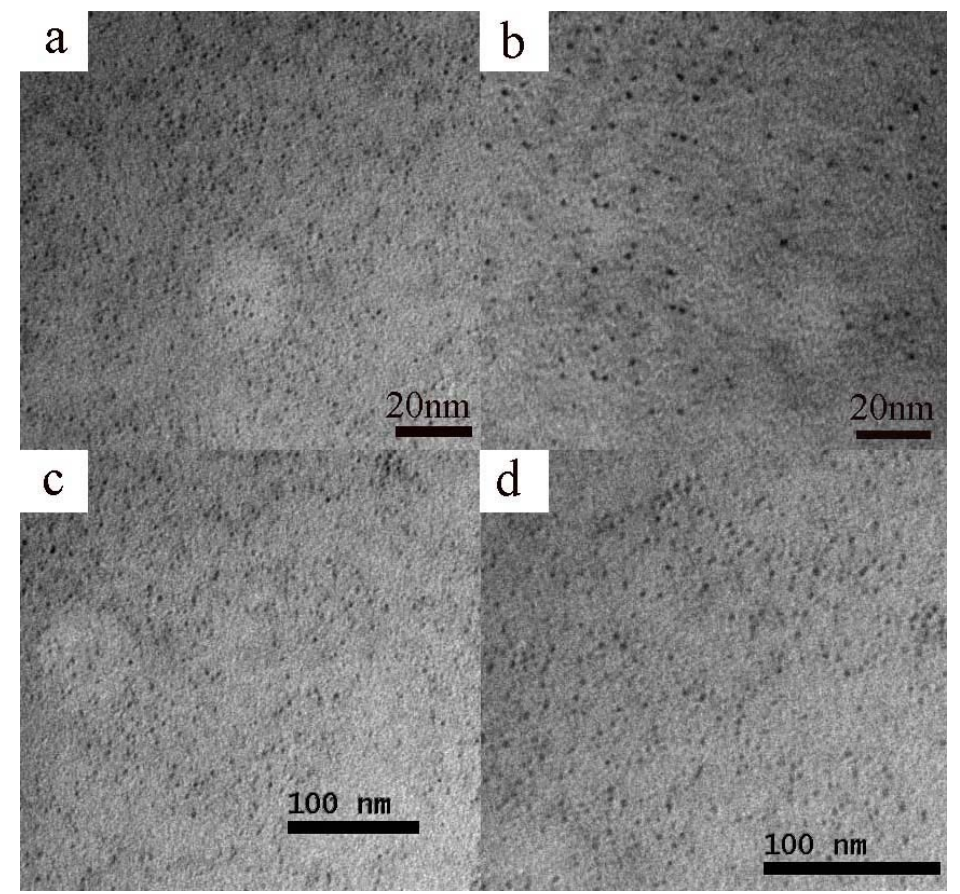

Figure S1 TEM images of SiQDs with different diameters: (a) 1 2 nm and (b) 3 4 nm; TEM images of partially oxidized SiQDs (c, 3 4 nm) and full oxidized SiQDs (d, 3 4 $\mathrm{nm})$. 


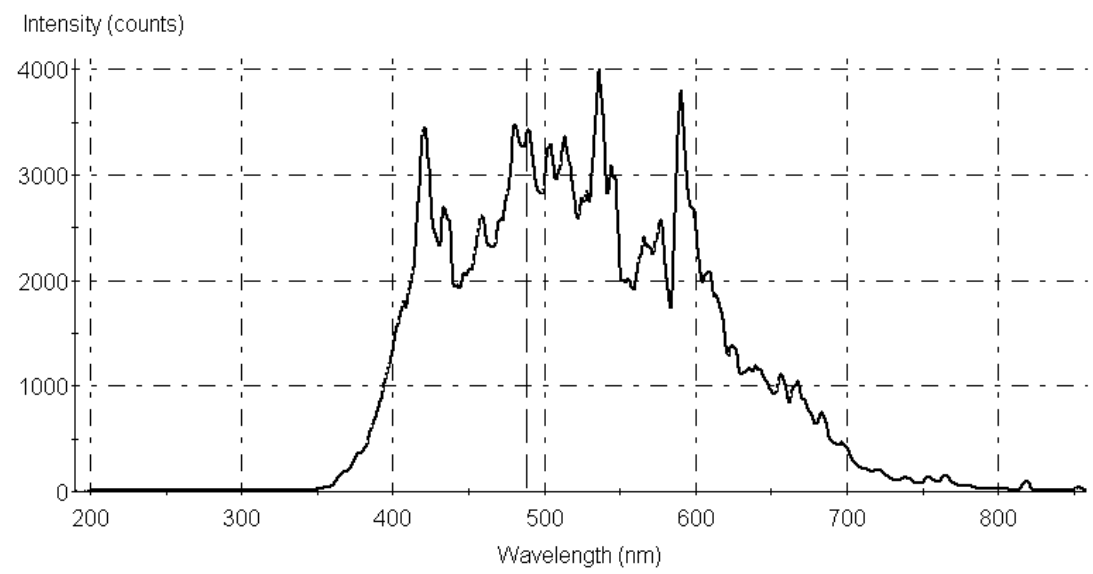

Figure $\mathrm{S} 2$ the spectrum of the halogen lamp.

\section{Photocatalytic reduction of $\mathrm{CO}_{2}$ in water or sodium carbonate $\left(\mathrm{Na}_{2} \mathrm{CO}_{3}\right)$}

$0.01 \mathrm{M}$ aqueous solution of $\mathrm{Na}_{2} \mathrm{CO}_{3}$ (Sigma-Aldrich, 99.99\%) was prepared in DI water. One piece of silicon wafer $(2 \mathrm{~cm} \times 10 \mathrm{~cm})$ coated with SiQDs was put into 100 $\mathrm{mL}$ of $\mathrm{Na}_{2} \mathrm{CO}_{3}$ solution. $\mathrm{CO}_{2}$ at a flow rate of $10 \mathrm{~mL} \cdot \mathrm{min}^{-1}$ was bubbled through the $\mathrm{Na}_{2} \mathrm{CO}_{3}$ solution. The reaction mixture was illuminated by a $150 \mathrm{~W}$ halogen lamp. Samples were collected every one hour from the mixture. To study the relationship between reduction rate and $\left[\mathrm{CO}_{3}{ }^{2-}\right]$ concentration, experiments were carried out for $12 \mathrm{~h}$ and the reduction rate was determined by the concentration of reduction product, i.e. formaldehyde and formic acid. The amount of formaldehyde was determined photospectrometrically by Nash reagent at $\lambda_{\max }=412 \mathrm{~nm}$. The concentration of formaldehyde in the sample was determined from a calibration curve. The concentration of formic acid was measured indirectly using Nash reagent by converting formic acid into formaldehyde by treatment of magnesium and hydrochloric acid. Other products were not measured.

Nash reagent was prepared by adding $150 \mathrm{~g}$ ammonium acetate, $3 \mathrm{~mL}$ acetic acid, and $2 \mathrm{~mL}$ redistilled acetyl acetone in $1 \mathrm{~L}$ water. The reagent was kept in the dark to avoid photochemical reaction. For measurement of the concentration of formaldehyde, the sample solution $(0.5 \mathrm{~mL})$ was taken in a test tube containing $5 \mathrm{~mL}$ of Nash reagent. The mixture was heated for $5 \mathrm{~min}$ in a water bath of $50-60^{\circ} \mathrm{C}$. The solution developed a bright yellow color, and after cooled to room temperature the optical intensity was measured at $\lambda_{\max }=412 \mathrm{~nm}$. The UV-visible spectrum of the product for the determination of formaldehyde by Nash reagent is shown in Figure S3. 


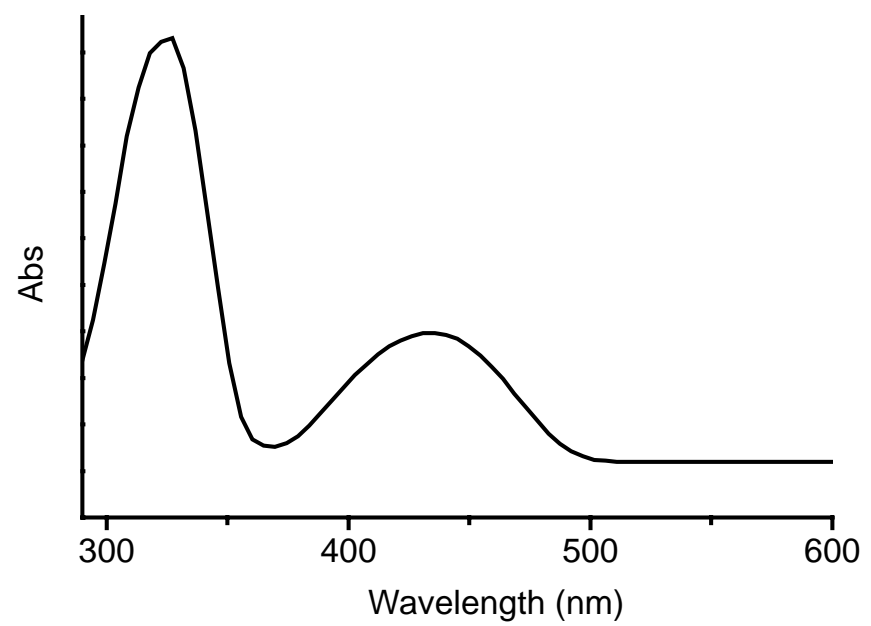

Figure S3 UV-visible spectrum of the product for the determination of formaldehyde by Nash reagent.

\section{$\square$. Photocatalytic decomposition of methyl red}

The reaction mixture of $20 \mathrm{~mL}$ SiQDs colloidal solution (SiQDs/n-hexane) and $100 \mathrm{~mL}$ methyl red solution (methyl red/DI water, about $2 \times 10^{-4} \mathrm{M}$ ) was illuminated by a $150 \mathrm{~W}$ halogen lamp. Samples were collected every minute from the system. The amount of methyl red was determined spectrophotometrically at $\lambda_{\max }=490 \mathrm{~nm}$. The UV-visible spectrum of the methyl red solution is shown in Figure S4.

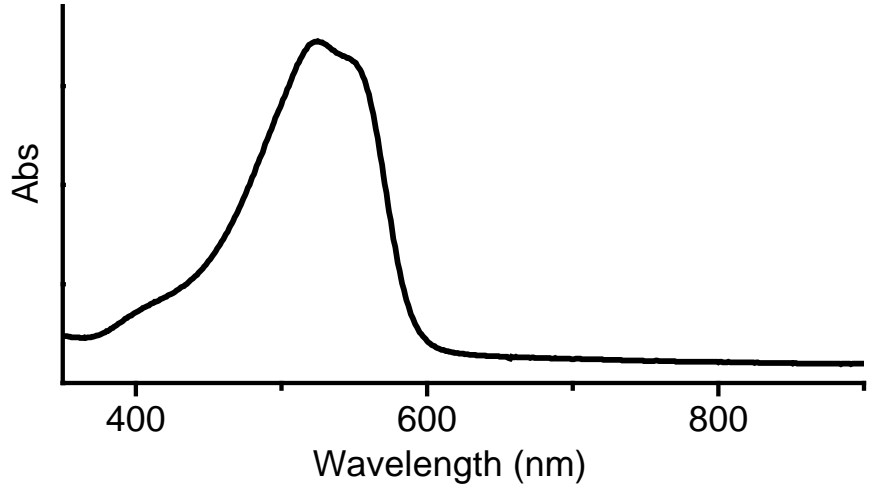

Figure S4 Ultraviolet-visible spectrum of the methyl red solution.

\section{$\square$. Photochemical conversion of benzene into phenol}

Aqueous-ionic liquid biphasic hydroxylation of benzene was carried out in a 100 $\mathrm{mL}$ round-bottomed flask equipped with a magnetic stirrer and a thermometer. SiQDs catalyst (suitable amount, $\sim 0.5 \mathrm{mg}$ ) was put into $50 \mathrm{~mL}$ benzene $(99.999 \%$ ) and $20 \mathrm{~mL}$ aqueous solution of $\mathrm{H}_{2} \mathrm{O}_{2}(10-15 \%)$. The resulting system was stirred under illumination of a $150 \mathrm{~W}$ halogen lamp for $12 \mathrm{~h}$ at ambient condition. Different samples were collected every 0.5 hours. At the end of the reaction, the aqueous and organic phases were separated. The product was analyzed by gas chromatograph. Benzene conversion was about $7 \%$. The conversion selectivity to phenol is about $\sim 100 \%$ based on benzene. 


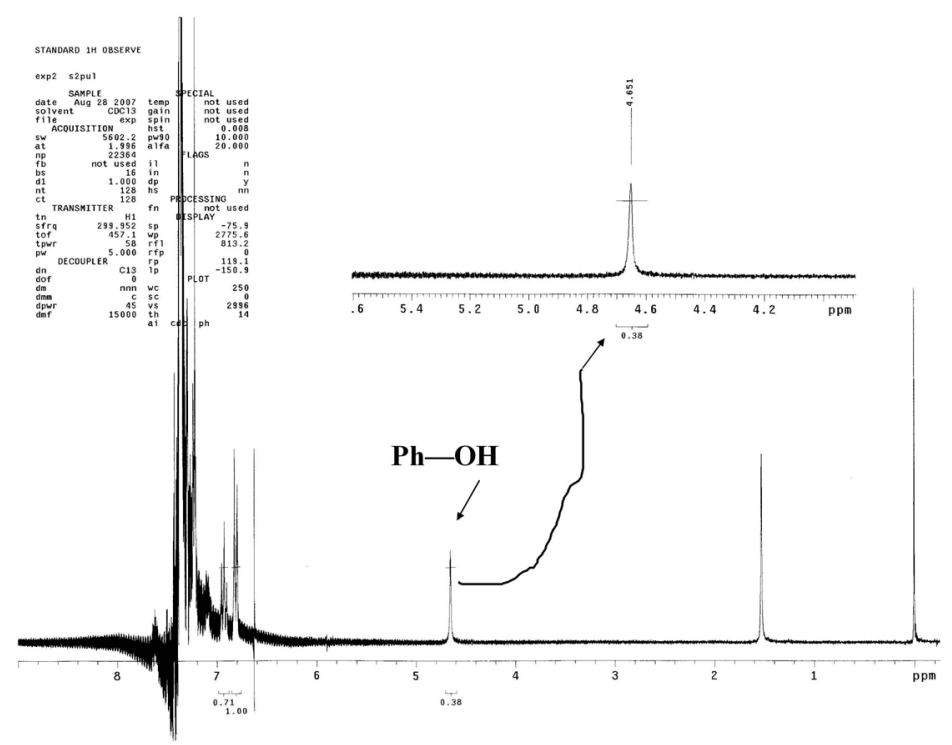

Figure S5 ${ }^{1} \mathrm{H}-\mathrm{NMR}$ of the products (phenol in benzene) with $\mathrm{CDCl}_{3}$ as solvent.

\section{Kinetic analysis of benzene to phenol}

Surface reaction is recognized as the major reaction pathway in the photochemical reaction. ${ }^{1-3}$ Oxidation of benzene on SiQDs surface typically proceeded via the sequence of dispersion, adsorption, surface reaction, and desorption. Dispersion was supposed to be the rate-determination step for the heterogeneous photocatalytic reaction by SiQDs. After elimination of the diffusion effect by vigorous stirring, the overall rate of photocatalytic reaction would be determined by surface reaction when the rate of adsorption and desorption is very high. In the reaction system (which is a mixture of an aqueous phase of $\mathrm{H}_{2} \mathrm{O}_{2}$ and an organic phase), since benzene is in high excess relative to $\mathrm{H}_{2} \mathrm{O}_{2}$, benzene concentration can be regarded as constant throughout the reaction. As a result, the coverage of benzene on SiQDs surface can also be regarded constant. Thus, the reaction rate ( $r$ ), adsorption and coverage of $\mathrm{H}_{2} \mathrm{O}_{2}$ on SiQDs surface are related as follows:

$$
\mathrm{r}=\mathrm{k} \theta_{\mathrm{A}} \theta_{\mathrm{B}} \theta_{\mathrm{Si}}
$$

Where $\mathrm{k}$ - Rate constant of surface reaction

$\theta_{\mathrm{A}}$ - Coverage of benzene on SiQDs surface

$\theta_{\mathrm{B}}$ - Coverage of $\mathrm{H}_{2} \mathrm{O}_{2}$ on SiQDs surface

$\theta_{\mathrm{Si}}$ - Coverage of surface active centers on SiQDs

$\theta_{\mathrm{Si}}$ and $\theta_{\mathrm{A}}$ can be regarded constant in the present system. When the product adsorption on SiQDs is weak, $\theta_{B}$ can be determined by Langmuir equation $\left(\theta_{B}\right.$ $\left.=K_{B}[B] /\left(1+K_{A} K_{B}[A][B]\right)\right)$. Substitution of $\theta_{B}$ into formula (1) yields

$$
1 / \mathrm{r}=1 / \mathrm{kK}_{\mathrm{B}} \bullet 1 /[\mathrm{B}]+1 / \mathrm{k} \bullet 1 /[\mathrm{B}]
$$

Where $\mathrm{K}_{\mathrm{B}}$-Adsorption equilibrium constant of $\mathrm{H}_{2} \mathrm{O}_{2}$ on SiQDs surface

[B]-Concentration of $\mathrm{H}_{2} \mathrm{O}_{2}$

Derivation of (2): $\quad \mathrm{r}=\mathrm{k} \theta_{\mathrm{A}} \theta_{\mathrm{B}} \theta_{\mathrm{Si}}$ and

$$
\mathrm{r}=\mathrm{k} \theta_{\mathrm{A}} \theta_{\mathrm{Si}} \mathrm{K}_{\mathrm{B}}[\mathrm{B}] /\left(1+\mathrm{K}_{\mathrm{A}} \mathrm{K}_{\mathrm{B}}[\mathrm{A}][\mathrm{B}]\right)
$$




$$
\mathrm{r}=\left(\mathrm{kK}_{\mathrm{B}}[\mathrm{B}]\right) /\left(1+\mathrm{K}_{\mathrm{B}}[\mathrm{B}]\right) \quad\left(\theta_{\mathrm{A}} \text { and } \mathrm{K}_{\mathrm{A}} \text { are constant }\right)
$$

The above formula is Langmuir-Hinshelwood dynamic equation, which shows the linear relationship of $1 / \mathrm{r}$ and $1 /[\mathrm{B}]$. Analysis of formula (2) leads to the following conclusions:

1. When $[B]$ is small and $K_{B}[B]<<1, \ln \left([B]_{0} /[B]\right)$ and $t$ (reaction time) will be linear; then the reaction is first order.

Derivation: $\quad \mathrm{r}=\left(\mathrm{kK}_{\mathrm{B}}[\mathrm{B}]\right) /\left(1+\mathrm{K}_{\mathrm{B}}[\mathrm{B}]\right)$

$\mathrm{r}=\mathrm{kK}_{\mathrm{B}}[\mathrm{B}]\left(1 /\left(1+\mathrm{K}_{\mathrm{B}}[\mathrm{B}]\right) \approx 1\right)$

Therefore $\quad r=-d[B] / d t=k K_{B}[B]$; here $t$ is the reaction time

$$
\begin{aligned}
& \int \mathrm{d}[\mathrm{B}] /[\mathrm{B}]=-\int_{\mathrm{kK}} \mathrm{dt} \\
& \ln [\mathrm{B}]=-\mathrm{kK}_{\mathrm{B}} \mathrm{t}+\mathrm{C}_{1} \quad\left(\mathrm{C}_{1} \text { : Integration constant }\right)
\end{aligned}
$$

2. When $[\mathrm{B}]$ is large, adsorption of $\mathrm{H}_{2} \mathrm{O}_{2}$ on catalyst surface reaches saturation, $\theta_{\mathrm{B}} \approx 1$ and $\left(1 / \mathrm{kK}_{\mathrm{B}} \bullet 1 /[\mathrm{B}]\right) \rightarrow 0,[\mathrm{~B}]$ and $\mathrm{t}$ are in linear relationship; then the reaction is zeroth order.

Derivation: $\quad 1 / \mathrm{r}=(1 / \mathrm{k}) \bullet\left(\left(1+\mathrm{K}_{\mathrm{B}}[\mathrm{B}]\right) /\left(\mathrm{K}_{\mathrm{B}}[\mathrm{B}]\right)\right)$

$$
\begin{array}{cl}
1 / \mathrm{r} & =(1 / \mathrm{k}) \cdot\left(1+\left(1 / \mathrm{K}_{\mathrm{B}}[\mathrm{B}]\right)\right) \\
\mathrm{r} & =\mathrm{k} \quad\left(1 / \mathrm{K}_{\mathrm{B}}[\mathrm{B}] \approx 0\right)
\end{array}
$$

Therefore $\quad \mathrm{r}=-\mathrm{d}[\mathrm{B}] / \mathrm{dt}=\mathrm{k}$

$$
\begin{aligned}
& \int \mathrm{d}[\mathrm{B}]=-\int \mathrm{kdt} \\
& {[\mathrm{B}]=-\mathrm{kt}+\mathrm{C}_{2} \quad\left(\mathrm{C}_{2} \text { : Integration constant }\right)}
\end{aligned}
$$

3. The reaction lies between zeroth and first order when $[\mathrm{B}]$ is at intermediate value.

Therefore, the Langmuir-Hinshelwood equation shows that the photocatalytic oxidation of benzene gradually changes from first order to zeroth order as the concentration of the reactant $\left(\mathrm{H}_{2} \mathrm{O}_{2}\right)$ increases.

\section{Reference}

(1)Bhatkhande, D.S.; Pangarkar, V.G.; Beenackers, A. J. Chem. Technol. Biotechnol. 2001, 77, 102.

(2)Kabra, K.; Chaudhary, R.; Sawhney, R.L. Ind. Eng. Chem. Res. 2004, 43, 7683.

(3)Linsebigler, A.L.; Lu, G.; Yates, J. T. Chem. Rev. 1995, 95, 735. 